\section{Parámetros productivos de cuyes (Cavia porcellus) suplementados con harina de sangre bovina}

\section{Production parameters of guinea pigs (Cavia porcellus) supple- mented with bovine bloodmeal}

\author{
Segundo Zamora' y Manuel Callacná
}

RESUMTEN

El presente estudio evaluó el efecto de la inclusión de diferentes niveles de harina de sangre (HS) de bovino (Bos taurus) procesada artesanalmente en los parámetros productivos de cuyes (Cavia porcellus) en etapa de crecimiento. Para lo cual, se emplearon 36 cuyes machos de 21 días de edad, que fueron ubicados en pozas y distribuidos aleatoriamente en cuatro grupos experimentales de 9 animales cada uno. Los animales fueron asignados a cuatro tratamientos que contenían 4 \%, 8 \% y 12 \% de HS, así como una dieta control que no contiene HS. Los resultados mostraron que al realizar la comparación entre análisis bromatológico con equipos tradicionales y uso de Espectroscopia de Infrarrojo Cercano (NIRS por sus siglas en ingles) en la harina de sangre, se encontraron valores en proteína cruda $75,76 \%$ y $72,14 \%$ respectivamente; extracto etéreo $1,03 \%$ y $1,96 \%$; cenizas $4,87 \%, 7,38 \%$ respectivamente. No se encontraron diferencias significativas entre el tratamiento control y los tratamientos con HS en relación al peso promedio final, ganancia diaria de peso vivo, consumo total acumulado de materia seca (MS), rendimiento de carcasa y peso de vísceras. La inclusión de $12 \%$ de HS mejoró la eficiencia económica en 1,39 soles más con respecto al grupo control. Además, la inclusión de HS a la alimentación de cuyes no tuvo ningún efecto negativo en el desempeño productivo y composición corporal, en cambio, mejoró la eficiencia económica y rentabilidad.

\section{ABSTRACT}

The present study was conducted to evaluate the effect of different levels of bovine blood meal (Bos taurus) processed in the productive parameters of guinea pigs (Cavia porcellus) in the growing stage. Thirty-six male guinea pigs of 21 days old were placed in cages and randomly distributed in four experimental groups of 9 animals each; animals were assigned to four treatments containing $4 \%, 8 \%$ and $12 \%$ of craft processed bovine blood meal (BM), as well as a control diet containing no HS. The results showed that when comparing between bromatological analysis and the use of Infrared Spectroscopy (NIRS) in blood meal, crude protein values were found in $75.76 \%$ and $72.14 \%$ respectively, ethereal extract $1.03 \%$ and $1.96 \%$, Ash: $4.87 \%$, 7.38 respectively. No significant differences were found between the control treatment and treatments with HS in relation to the final average weight, daily gain of live weight, cumulative total dry matter (DM) consumption, carcass yield, viscera weights. The inclusion of $12 \%$ HS improved economic efficiency by 1.39 soles more than the control group. The results of this study revealed that the inclusion of $12 \%$ of blood meal in guinea pigs had no negative effect on productive performance and body composition, but did improve economic efficiency and profitability.

\author{
Phlabras clave \\ cuyes $\cdot$ harina de sangre \\ alimentación · parámetros
}

\author{
KEYWORDS \\ guinea pig $\cdot$ blood meal \\ feeding $\cdot$ parameter
}

\section{INTRODUCCIÓN}

En el Perú, la producción de cuyes es una alternativa de solución a los problemas alimentarios en los estratos sociales más bajos porque el consumo de esta carne aporta proteínas de alto valor biológico en la dieta familiar. Además, la crianza genera ingresos y permite, en el corto plazo, producir gran-
\end{abstract}

des volúmenes de carne en espacios reducidos. El uso de alimentos balanceados es aún incipiente y los pocos productores que la usan recurren a insumos de tipo proteico que provienen de la costa como harina de pescado o importados como harinas de soya, cuyos costos son altos.

La harina de sangre es un subproducto de la indus-

'Ingeniero Zootecnista. Investigador del IGBI y docente FIZAB-UNTRM. Correo Electrónico:josé.zamora@untrm.edu.pe
${ }^{2}$ Ingeniero Zootecnista. Docente de la Universidad Nacional de Trujillo. Correo Electrónico: mcallacna@unitru.edu.pe 
tria de carnes, obtenida por la desecación de la sangre, se caracteriza por el alto contenido de proteína y una alta digestibilidad. Los centros de sacrificio (camales) desperdician la sangre y el contenido ruminal, sin aprovechar esta valiosa fuente proteica para la utilización en dietas de animales (FEDNA, 2005). La escasez actual de proteína animal en los países subdesarrollados justifica la investigación del potencial de algunos recursos producidos localmente para animales, tales como harina de sangre e incluirla en dietas con la finalidad de mejorar el margen de ganancias mediante la reducción del uso de las fuentes convencionales como soya (Glycine max) (D'Mello 1995). El presente experimento fue conducido para probar la hipótesis de que la adición de harina de sangre procesada artesanalmente en dietas de cuyes mejora los índices productivos.

\section{MATERIALES Y MÉTODOS \\ Ubicación}

El presente estudio se realizó en el Módulo de Investigación en animales menores, perteneciente a la Estación Experimental de Chachapoyas de la Facultad de Ingeniería Zootecnista, Agronegocios y Biotecnología (FIZAB) en la Universidad Nacional Toribio Rodríguez de Mendoza (UNTRM) Chachapoyas, Amazonas, Perú. Los animales fueron instalados en pozas con un sistema de ventilación (temperaturas en el módulo entre $19-25^{\circ} \mathrm{C}$ ). Los cuyes fueron expuestos a un fotoperiodo natural (12-13 horas).

Animales, tratamientos y diseño experimental Un total de 36 cuyes machos de raza Perú (21 días de edad y $375 \mathrm{~g}$ de peso promedio inicial) fueron alojados en 12 pozas de $40 \times 40 \times 120 \mathrm{~cm}$ cada una. Los cuyes fueron distribuidos aleatoriamente en 4 tratamientos iguales con 9 animales cada uno; los animales fueron asignados a cuatro tratamientos conteniendo $4 \%$, $8 \%$ y $12 \%$ de HS; y un grupo control sin HS. Todos los animales en promedio recibieron alimento en forma de harina en cantidad de 45 gramos de concentrado y $110 \mathrm{~g}$ de forraje maíz por cuy al día, además de agua ad libitum. Dentro de la composición de alimento balanceado se aplicó promotor de crecimiento. La composición y el análisis químico de las dietas experimentales se muestran en la Tabla 1. Los animales fueron pesados individualmente de manera semanal durante las 7 semanas que duró el periodo experimental para determinar parámetros como consumo de alimento, ganancia de peso corporal y conversión alimenticia. No hubo enfermedades y el índice de mortalidad fue cero durante la investigación.

Tabla 1. Composición y análisis químico de las dietas experimentales

\begin{tabular}{|c|c|c|c|c|}
\hline Dietas (\%) & Control & $4 \%$ HS & $8 \% \mathrm{HS}$ & $12 \% \mathrm{HS}$ \\
\hline \multicolumn{5}{|l|}{ Ingredientes } \\
\hline Harina de sangre & 0,0 & 4,30 & 8,57 & 12,79 \\
\hline Maíz molido & 39,88 & 42,90 & 45,94 & 48,96 \\
\hline Harina de soya & 23,57 & 16,38 & 9,25 & 2,17 \\
\hline Subproducto de trigo & 19,71 & 19,62 & 19,52 & 19,44 \\
\hline Subproducto de arroz & 9,85 & 9,81 & 9,77 & 9,72 \\
\hline Melaza de caña & 4,73 & 4,71 & 4,69 & 4,67 \\
\hline Carbonato de calcio & 1,65 & 1,67 & 1,70 & 1,73 \\
\hline Sal común & 0,41 & 0,39 & 0,33 & 0,28 \\
\hline Pre mezcla vit \& min. & 0,12 & 0,12 & 0,12 & 0,12 \\
\hline Metionina & 0,08 & 0,10 & 0,11 & 0,12 \\
\hline TOTAL & 100 & 100 & 100 & 100 \\
\hline \multicolumn{5}{|c|}{ Análisis químico (g/kg en base a materia seca) } \\
\hline Energía digestible (Mcal/kg) & 309,0 & 312,0 & 315,0 & 318,0 \\
\hline Proteina cruda & 185,0 & 185,0 & 185,0 & 185,0 \\
\hline FDN & 187,0 & 188,0 & 189,0 & 190,0 \\
\hline FDA & 98,0 & 99,0 & 100,0 & 101,0 \\
\hline Extracto etéreo & 37,2 & 38,1 & 39,0 & 39,9 \\
\hline Calcio & 8,5 & 8,5 & 8,5 & 8,5 \\
\hline Fósforo total & 6,4 & 6,2 & 5,9 & 5,6 \\
\hline Metionina & 4,0 & 4,0 & 4,0 & 4,0 \\
\hline Lisina & 9,3 & 10,4 & 11,5 & 12,5 \\
\hline
\end{tabular}


Procesamiento de harina de sangre y elaboración de dietas alimenticias.

La harina de sangre se elaboró siguiendo la metodología propuesta por Cifuentes (2007). Luego de obtenida fue almacenada a una temperatura menor a $10^{\circ} \mathrm{C}$ y fue usada para formular y combinar con un mezclador vertical de 300 kilos por batch. El análisis químico de la harina de sangre se muestra en la tabla 2.

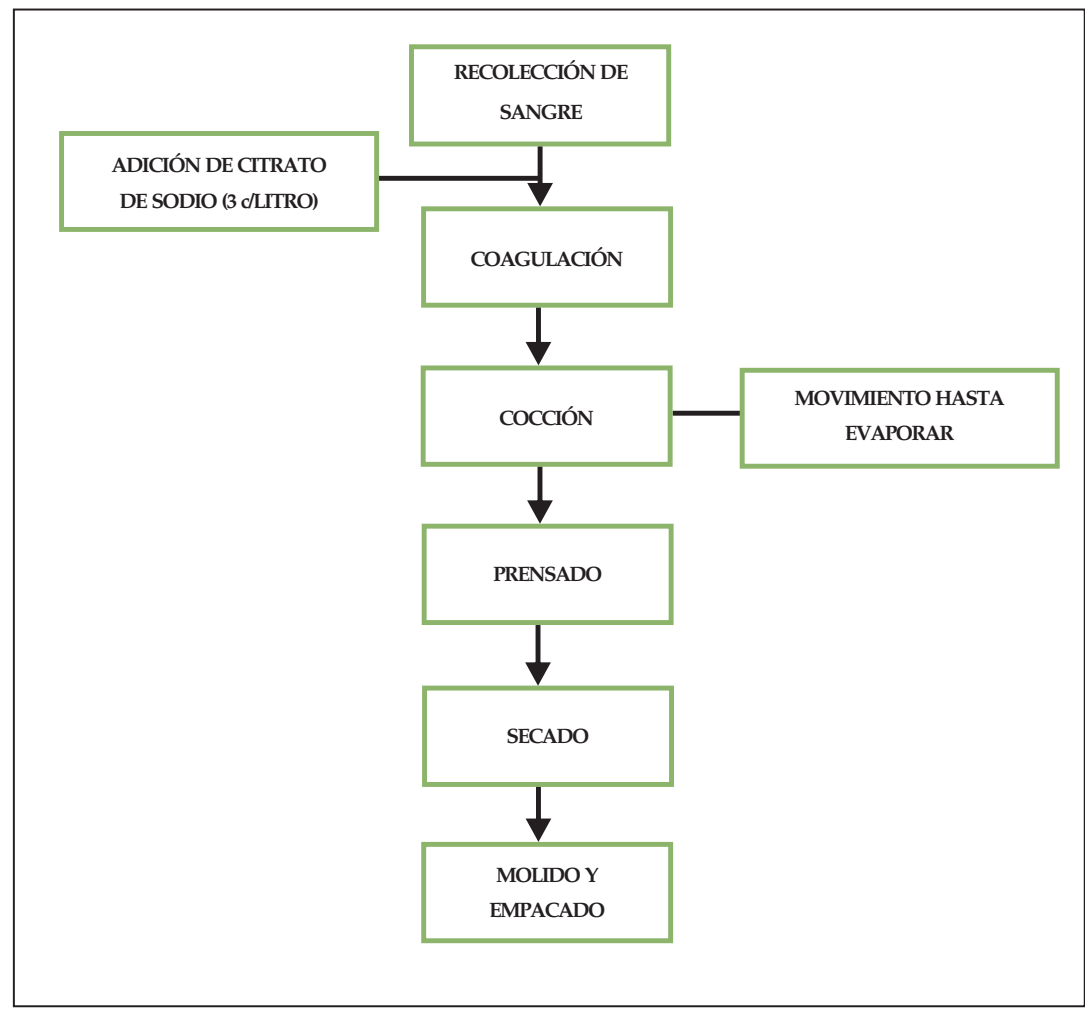

Figura 1. Flujograma de proceso de harina de sangre, modificado de Cifuentes (2007)

\section{Composición química}

La composición química de la harina de sangre fue determinada con los procedimientos estandarizados según AOAC (2000) para determinar materia seca, ceniza, proteína cruda y extracto etéreo. Además, la harina de sangre fue evaluada con el equipo de Espectroscopia de Infrarrojo cercano (NIRS en inglés) para realizar la comparación con la metodología química.

\section{Carcasa y evaluación de órganos}

Al final del periodo experimental, 3 cuyes con seis horas de ayuno y $956 \mathrm{~g}$ de peso vivo fueron seleccionados de cada tratamiento, luego cada cuy fue beneficiado cortando la vena yugular con cuchillo filudo basado en el método "Halal". Se tomaron todos los cuidados para evitar sufrimiento innecesario a los animales durante el beneficio, a partir la metodología propuesta por López (2004). Se anotó el peso al beneficio cuando se desangraron completamente. La carcasa vacía, corazón, pulmón, hígado, fueron separadas y pesadas.

\section{Eficiencia económica}

La eficiencia económica para el alimento se calculó a partir de la siguiente ecuación (Abdella et al., 1988).
Eficiencia económica $(\%)=\frac{\text { Ingreso neto }}{\text { CT Alim }} \times 100$

Donde:

Ingreso Neto $=$ Precio de venta - Costo de alimentación

Precio de venta $(P V)=$ Precio por kilo de peso $x$

Costo total

Ganancia total de peso

por alimento $(\mathrm{CT}$ alim. $)=$ Consumo promedio de alimento $x$ precio por kilo de alimento

\section{Análisis estadístico}

Se utilizó un diseño completamente al azar (DCA). Los datos fueron analizados mediante el Análisis de varianza (ANVA) al $5 \%$ de significancia. Se utilizó la prueba de Tukey para determinar las diferencias entre las medias de los tratamientos. 


\section{RESULTADOS}

Comparación de composición química de harina de sangre

Los datos de la comparación de composición química de la harina de sangre se muestran en la tabla 2 , indicando no ser significativo entre los valores que arrojan el análisis químico proximal vs Espectroscopia de Infrarrojo Cercano (NIRS).

Tabla 2. Comparación valor nutritivo harina de sangre

\begin{tabular}{lcc}
\hline Ítems & HS a & HS b \\
\hline Materia seca (\%) & 82,13 & 79,56 \\
Proteína cruda (\%) & 75,76 & 72,14 \\
Fibra Cruda (\%) & 1,11 & 0,77 \\
Extracto Etéreo (\%) & 1,03 & 1,96 \\
Cenizas (\%) & 4,87 & 7,38 \\
Extracto Libre Nitrógeno (\%) & 17,23 & \\
Energía Bruta (Kcal/Kg MS) & 5138,65 & \\
\hline
\end{tabular}

${ }^{a}$ Laboratorio de Nutrición Animal - Universidad Nacional de Cajamarca (2014).

${ }^{\mathrm{b}}$ Espectroscopia de reflectancia del infrarrojo cercano (NIRS) Equipo Spectra Star Feed Analyzer UNITY Scientific. Universidad Nacional Toribio Rodríguez de Mendoza de Amazonas.

\section{Características de rendimiento}

Los resultados del rendimiento productivo en cuyes alimentados con harina de sangre se presentan en la tabla 3. Todos los cuyes empezaron con un peso inicial similar $(375,25 \mathrm{~g}$ en promedio). Los cuyes que recibieron T3 (12\% HS) alcanzaron el más alto peso vivo final, mientras que T1 ( $4 \% \mathrm{HS}$ ) reportaron el menor peso vivo final con un $8 \%$ menos que el T3, sin ninguna diferencia significativa $(p=0,527)$. El valor más alto para ganancia de peso corporal se logró con el T3 (12\% HS), mientras que el T1 (4 \% HS) tuvo el menor valor con un $10 \%$ menor, sin diferencias significativas $(p=0,553)$. No hubo diferencias significativas en relación al consumo de alimento $(p=0,375)$ entre los tratamientos. No hubo diferencias significativas entre los tratamientos para conversión alimenticia $(\mathrm{p}=0,38)$.

Tabla 3. Peso corporal y características de consumo de alimento de cuyes en crecimiento

\begin{tabular}{|c|c|c|c|c|c|}
\hline \multirow{2}{*}{ Ítems } & \multirow{2}{*}{ Control } & \multicolumn{3}{|c|}{ Niveles de harina de sangre } & \multirow{2}{*}{ p-valor } \\
\hline & & $4 \%$ & $8 \%$ & $12 \%$ & \\
\hline Peso vivo inicial $(\mathrm{g})$ & 376,44 & 365,55 & 380,33 & 378,67 & 0,904 \\
\hline Peso vivo final (g) & 1017,56 & 974,11 & 1031,44 & 1058,13 & 0,527 \\
\hline Ganancia de peso corporal (g/cuy/día) & 10,18 & 9,66 & 10,34 & 10,79 & 0,553 \\
\hline Consumo de alimento (g/cuy/día) & 66,09 & 63,73 & 64,01 & 62,41 & 0,375 \\
\hline $\begin{array}{l}\text { Conversión alimenticia } \\
\text { (g alimento/g ganancia) }\end{array}$ & 6,49 & 6,60 & 6,19 & 5,79 & 0,38 \\
\hline
\end{tabular}

\section{Características de carcasa}

Los resultados de las características de carcasa para cuyes en crecimiento conteniendo diferentes niveles de harina de sangre, se muestra en la tabla 4. No hubo diferencias significativas entre pesos de animales antes del sacrificio (ayunados) entre los tratamientos. Los porcentajes rendi- mientos de carcasa variaron entre T1 (4\% HS) con $71,51 \%$ con su valor más bajo y T3 (12\% HS) con $73,72 \%$ su valor más alto, sin ninguna diferencia significativa $(p=0,648)$. Finalmente, para el peso de vísceras no se encontraron diferencias significativas entre los tratamientos $(p=0,648)$.

Tabla 4. Características de carcasa en cuyes de crecimiento alimentos con diferentes niveles de harina de sangre $n=9$ cuyes por tratamiento.

\begin{tabular}{lccccc}
\hline \multirow{2}{*}{ Ítems } & \multirow{2}{*}{ Control } & \multicolumn{3}{c}{ Niveles de harina de sangre } & \multirow{2}{*}{ p-valor } \\
\cline { 3 - 5 } & & $\mathbf{4} \%$ & $\mathbf{8} \%$ & $\mathbf{1 2} \%$ & \\
\hline Peso vivo ayunado $(\mathrm{g})$ & 953 & 911,33 & 967,22 & 995 & 0,516 \\
Rendimiento carcasa (\%) & 73,67 & 71,51 & 72,91 & 73,72 & 0,648 \\
Peso de vísceras (g) & 177,50 & 143,00 & 163,25 & 159,00 & 0,648 \\
\hline
\end{tabular}

\section{Evaluación económica}

La evaluación económica de las dietas experimentales se presenta en la tabla 5. La inclusión de hari- na de sangre en las dietas experimentales en cuyes de crecimiento resultó en una marcada reducción de costo/kg de las dietas que se reflejaron en una 
mejor eficiencia económica de dichos tratamientos comparados con el grupo control. La relativa eficiencia económica fue superior para el T3
(12\%HS) que el Control (108\%), seguido por T2 (8 $\%$ HS) con $45 \%$ y finalmente T1 (4\% HS) con $17 \%$.

Tabla 5. Análisis del costo para cuyes en crecimiento

\begin{tabular}{lcccc}
\hline \multirow{2}{*}{ Ítems } & \multirow{2}{*}{ Control } & \multicolumn{3}{c}{ Niveles de harina de sangre } \\
\cline { 3 - 5 } & & $\mathbf{4} \%$ & $\mathbf{8} \%$ & $\mathbf{1 2} \%$ \\
\hline Costo total por alimentación/cuy & 4,35 & 3,85 & 3,67 & 3,13 \\
Ganancia total de peso (kg) & 641,12 & 608,56 & 651,11 & 679,46 \\
Precio por kg de peso vivo & 20,00 & 20,00 & 20,00 & 20,00 \\
Precio de venta & 12,82 & 12,17 & 13,02 & 13,59 \\
Ingreso neto & 8,47 & 8,32 & 9,35 & 10,46 \\
Eficiencia económica & 1,95 & 2,16 & 2,55 & 3,34 \\
\hline Eficiencia económica relativa & 100,0 & 110,90 & 130,84 & 171,57 \\
\hline
\end{tabular}

\section{DISCUSIÓN}

Composición química de harina de sangre

Huarcaya (1987) muestra valores de PC de 65,6 $\%$, valor inferior al encontrado en esta investigación de 75,76 \%, debido posiblemente a métodos de preparación y tipo de secado. Sin embargo, estos resultados coinciden con los encontrados por Rosales-Conde y Tony (2006) PC (80,47 \%), Grasa (0,33\%), Ceniza (2,78\%).

\section{Características de rendimiento}

Respecto al incremento de peso vivo, se lograron ganancias con el T3 (12 \% HS) de 10,79 g, las mismas que difieren con Espinoza (1991), quien encontró valores menores de 5,5 g diario para las mismas cantidades de HS. Asimismo, Aquino (1991) encontró ganancias de peso diario con 5,42 g para un uso de $15 \%$ de harina de sangre. En los pesos finales, se encontraron pesos a las 9 semanas de 1058,13 g, esto difiere con Astete et al. (1991) quien encontró valores menores a los 75 días con $1044 \mathrm{~g}$.

El mayor consumo de alimento fue de $66,09 \mathrm{~g}$ (control) de materia seca al día, valor inferior al encontrado por Alviar y Cook (1985) de 89,7 g/día; así mismo Orellana et al. (2004) encontró valores de $74 \mathrm{~g}$ / día en un periodo de evaluación de 55 días.

La mejor conversión alimenticia a los 63 días obtenida con T3 (12\% HS) fue de 5,79, no se relaciona con la investigación de Orellana et al. (2004), quien encontró mayores valores con 7,07 (20\% HS) y 8,19 (sin harina de sangre). Asimismo, Chauca et al. (2006) evidencia valores de conversión mucho menor que están entre 2,68 a los 56 días de edad, debido probablemente a la falta en mejora genética de cuyes.

\section{Características de carcasa}

En el rendimiento de carcasa, se encontró valores de 73,72\% (12\% HS), el más alto valor. Además de ser superior al encontrado por Orellana et al. (2004) quien encontró valores de 67,99 \% en dietas que contienen $20 \%$ HS. Asimismo, resultados similares reporta Montes (2012) con rendimientos de $69,7 \%$.

Respecto a valores de composición química de la carne, Chauca et al. (1995) encontró valores de $75,25 \%$ y 20,3 \% para humedad y proteína respectivamente, resultados similares a los encontrados en la presente investigación en humedad $(65,6 \%)$ y proteína $(20,1 \%)$.

\section{CONCLUSIONES}

Al margen de los resultados económicos favorables con el uso de harina de sangre en las dietas de cuyes en crecimiento, para su uso, se debe tener un cuidado especial en la técnica de elaboración. Por ello, en la presente investigación no hubo diferencias significativas $(\mathrm{p}>0,05)$ en parámetros productivos como ganancias de peso, consumo de alimento y conversión alimenticia, demostrando que su uso en $12 \%$ reduce notablemente los costos de alimentación porque se obtuvo un beneficio de 1,39 soles más que el grupo control (equivalente $71,57 \%$ más).

\section{REFERENCIAS BIBLIOGRÁFICAS}

Abdella, H. M., Shalash, S. M. M., Boulos, N. Z., \& Selm, A. D. (1988). Effect on growing rabbits of feeding different levels of crude protein. The Journal of Applied Rabbit Research, 11, 252-256.

AOAC (Association of Official Analytical Chemists). (1990). Official methods of analysis. Assoc Anal Chem.

Alviar, D., \& Cook, F. (1985). Raciones para la gestación, lactación y crecimiento en cuyes mejorados 2,750 msnm Octava reunión científica anual de la Asociación Peruana 
de Producción Animal; resúmenes. Reunión científica anual, 8. Huancayo, 12-16 Nov. 1985.

Aquino, A. (1991). Uso de diferentes niveles de harina de sangre en la alimentación de cuyes. [Resumen]. XIV Reunión APPA. Cerro de Pasco, Perú.

Astete, P., Castro, J., \& Chirinos, D. (1991). Uso de harina de sangre cruda y cocida en el engorde de cuyes [Resumen]. XIV Reunión APPA. Cerro de Pasco, Perú.

Chauca, L., Higaona, R., Muscari, R., \& Pinto, J. (2006). Caracterización de la carcasa de seis genotipos de cuyes. Reunión Anual de la Asociación Peruana de Producción Animal. Huancayo: APPA.

Chauca L; Rosa H; Juan M. (1995). Caracterización de la carcasa de seis genotipos de cuyes. Instituto de Investigación y Extensión AgrariaCosecha Urbana CIP.

Cifuentes O. (2007). Proceso artesanal de producción de harina de sangre de bovino. www.engormix.com

D'Mello, J. P. F. (1995). Leguminous leaf meals in non-ruminant nutrition.

Espinoza, T. (1991). Engorde de cuyes castrados a base de concentrado y forraje. XIV Reunión APPA. Cerro de Pasco: Asociación Peruana de Producción Animal.

FEDNA (Fundación Española para el desarrollo de la nutrición animal) (2013) Disponible en http://www.fedna.com

FEDNA (Fundación Española para el desarrollo de la nutrición animal). (2005). Subproductos animales. Disponible en http:/ /www.fedna.com

Huarcaya, H.A. (1987). Valor Nutricional de la harina de sangre de broilers en el trópico. Tesis Ingeniero Zootecnista. Tingo María (Perú): Universidad Nacional Agraria de la Selva. 59 pp.

INIA.(2012). Tecnología en cuyes. Consultado el 10 de Junio de 2015. Disponible en www. Inia.gob.pe/tecnologías/crianzas.

López, R. (2004). Tecnología de mataderos (No. 04; TS1960, L6.).

Montes, I. T. (2012). Guía técnica: Asistencia técnica dirigida a la crianza tecnificada de cuyes. UNALM. Agrobanco. OAEPS. Cajabamba-Cajamarca, Perú.

Orellana, T. , Castro B., \& Chirinos, D. (2004). Harina de sangre de asnos como sustituto de harina de pescado en suplementos de cuyes en crecimiento. Facultad de Zootecnia. Universidad Nacional de Centro de
Perú Huancayo Perú.

Rosales-Conde, J. M., \& Tony, T. A. N. G. (2006). Composición química y digestibilidad de insumos alimenticios de la zona de Ucayali. Folia Amazónica, 8(2), 13-27. 